\title{
Port-Hamiltonian Based Control of the Sun-Earth 3D Circular Restricted Three-Body Problem: Stabilization of the $L_{1}$ Lagrange Point
}

\author{
Haotian Yan \\ Community School of Naples, Virginia, USA \\ Email: yhtbaoyuan@163.com
}

How to cite this paper: Yan, H.T. (2020) Port-Hamiltonian Based Control of the Sun-Earth 3D Circular Restricted Three-Body Problem: Stabilization of the $L_{1}$ Lagrange Point. Modern Mechanical Engineering, 10, 39-49.

https://doi.org/10.4236/mme.2020.103005

Received: September 3, 2019

Accepted: August 28, 2020

Published: August 31, 2020

Copyright $\odot 2020$ by author(s) and Scientific Research Publishing Inc. This work is licensed under the Creative Commons Attribution International License (CC BY 4.0).

http://creativecommons.org/licenses/by/4.0/

\begin{abstract}
In this paper, we use Port-Hamiltonian framework to stabilize the Lagrange points in the Sun-Earth three-dimensional Circular Restricted Three-Body Problem (CRTBP). Through rewriting the CRTBP into Port-Hamiltonian framework, we are allowed to design the feedback controller through energy-shaping and dissipation injection. The closed-loop Hamiltonian is a candidate of the Lyapunov function to establish nonlinear stability of the designed equilibrium, which enlarges the application region of feedback controller compared with that based on linearized dynamics. Results show that the Port-Hamiltonian approach allows us to successfully stabilize the Lagrange points, where the Linear Quadratic Regulator (LQR) may fail. The feedback system based on Port-Hamiltonian approach is also robust against white noise in the inputs.
\end{abstract}

\section{Keywords}

Port-Hamiltonian, Lagrange Points, Circular Restricted Three-Body Problem (CRTBP), Linear Quadratic Regulator (LQR)

\section{Introduction}

The Circular Restricted Three Body Problem (CRTBP) [1] has been widely studied by physicists, astronomers, and astrodynamics. This problem is focused on overcoming the difficulties in finding a suitable orbit for satellites. In the system of the CRTBP, there exist five equilibrium positions, which are known as Lagrange points. These Lagrange points are of scientific and engineering interest; e.g., artificial spacecrafts have been placed at the $L_{1}$ and $L_{2}$ Lagrange points with respect to the Sun and the Earth [2] [3] [4] [5] and Lagrange points with respect 
to the Earth and the Moon [6] [7].

However, that is also of the most demanding challenge; i.e., among the five Lagrange points, three of them are unstable [1] [8]. Fortunately, around these unstable points $\left(L_{1}, L_{2}, L_{3}\right)$, there exist stable halo orbits, which allow artificial satellite to reside. In order to design an orbit around Lagrange points, the most broadly used methods are based on linearization around a halo orbit or Lagrange point [9] [10] [11] [12] [13].

However, stabilization of a linear system is only able to guarantee the stability of the system against an infinitely small perturbation, but is unable to guarantee the stability against a finite disturbance. Disturbances for the Earth-Moon Lagrange points mission under CRTBP model include the eccentricity of the EarthMoon orbit, the gravity from the other bodies [14] and the solar radiation pressure. For the Sun-Earth Lagrange points mission, the perturbation from the Moon is critical. Lyapunov [15], in the year of 1895, proposed the Lyapunov method to establish the stability of nonlinear system. The difficulties of Lyapunov's method is finding a Lyapunov function to establish the stability. For Hamiltonian system, the conserved quantity Hamiltonian is a candidate of Lyapunov function to establish the stability of the system. The Jacobian integral [16], which is the conserved quantity for the CRTBP in a rotating coordinate, allows us to formulate the CRTBP as the Hamiltonian system.

Port-Hamiltonian system [17] [18] generalizes the Hamiltonian system and allows us to take input and dissipation into consideration, which are described as "port". This framework has the potential to model, analyze and especially, control complex physical systems and their interconnections [17]. The Port-Hamiltonian framework also provides a physics-based control strategy [19]-[24], which focuses on shaping the closed-loop Hamiltonian as the candidate of Lyapunov function.

In this research, we use the Port-Hamiltonian system to reformulate the SunEarth three-dimensional CRTBP, which retains the original nonlinear dynamics in contrast to the linear approximation. Then, we designed the feedback control law to stabilize the unstable Lagrange points by taking input and dissipation as two other actors into consideration. The closed-loop Hamiltonian serves as the candidate of Lyapunov's function, which helps to establish the nonlinear stability of the open-loop unstable Lagrange points. This research provides further possibility in Lagrange point launching mission.

\section{The Circular Restricted Three Body Problem}

\subsection{Governing Equations}

In Circular Restricted Three Body Problem (CRTBP), it requires the largest two objects of the three have a significantly larger mass compared to the third one; i.e., $M_{1} \gg M_{3}$ and $M_{2} \gg M_{3}$. The largest two objects are in circular orbits centered at their center of mass. We consider the third object, $M_{3}$, moving in three-dimensional (3D) space; i.e., 3D-CRTBP. In order to solve this problem, 
we need to use mathematical method to approximate this situation. We set the center of mass of the system as the origin and we set the position of $M_{1}$ at $(-\mu, 0)$, position of $M_{2}$ at $(1-\mu, 0)$, where we define $\mu=M_{2} / M$ and $M=M_{1}+M_{2}$. Then, the dynamics of the third body can be described as [1]:

$$
\begin{gathered}
\frac{\mathrm{d}^{2} x}{\mathrm{~d} t^{2}}-2 \frac{\mathrm{d} y}{\mathrm{~d} t}=x-(1-\mu) \frac{x+\mu}{r_{1}^{3}}-\mu \frac{x-1+\mu}{r_{2}^{3}} \\
\frac{\mathrm{d}^{2} y}{\mathrm{~d} t^{2}}+2 \frac{\mathrm{d} x}{\mathrm{~d} t}=y-(1-\mu) \frac{y}{r_{1}^{3}}-\mu \frac{y}{r_{2}^{3}} \\
\frac{\mathrm{d}^{2} z}{\mathrm{~d} t^{2}}=-\left(\frac{1-\mu}{r_{1}^{3}}-\frac{\mu}{r_{2}^{3}}\right) z
\end{gathered}
$$

where

$$
\begin{gathered}
r_{1}=\sqrt{(x+\mu)^{2}+y^{2}+z^{2}} \\
r_{2}=\sqrt{(x-1+\mu)^{2}+y^{2}+z^{2}}
\end{gathered}
$$

We set $\mu=3.003490055444426 \times 10^{-6}$, which is the mass ratio in the Sun-Earth CRTBP system (see Table 1).

\subsection{The Lagrange Points and the Jacobian Integral}

Lagrange points are points in systems on which the third object could reach equilibrium state. According to [1], there exists five Lagrange points in the CRTBP. Three of the five points, $L_{1}, L_{2}$, and $L_{3}$ can be computed using algebraic equations:

$$
\begin{gathered}
x-(1-\mu) \frac{x+\mu}{r_{1}^{3}}-\mu \frac{x-1+\mu}{r_{2}^{3}}=0 \\
y=0
\end{gathered}
$$

In CRTBP, Jacobian integral is the only conserved variable, which is widely used to derive solutions in special cases, and is expressed as what follows in the $(x, y)$-coordinate system:

$$
C=\left(x^{2}+y^{2}\right)+2\left(\frac{1-\mu}{r_{1}}+\frac{\mu}{r_{2}}\right)-\left(x^{2}+y^{2}+z^{2}\right)
$$

Conservation of energy (kinetic energy and potential energy) is shown by Jacobian integral in CRTBP under synodic coordinates description, in which the dissipation caused by the air drag is not included in the model.

Table 1. Lagrange points for the Sun-Earth CRTBP with $\mu=3.003490055444426 \times 10^{-6}$.

\begin{tabular}{cccccc}
\hline & $L_{1}$ & $L_{2}$ & $L_{3}$ & $L_{4}$ & $L_{5}$ \\
\hline$x$ & 0.990026583427689 & 1.010034125978081 & 1.000001251454178 & 0.499996996509945 & 0.499996996209945 \\
$y$ & 0 & 0 & 0 & 0.866025403784439 & 0.866025403784439 \\
\hline
\end{tabular}




\section{Port-Hamiltonian System}

\subsection{Reformulation of CRTBP}

If we use the Hamiltonian proportional to the Jacobian integral:

$$
H=-\frac{C}{2}=\frac{1}{2}\left(\dot{x}^{2}+\dot{y}^{2}+\dot{z}^{2}\right)-\frac{1}{2}\left[2\left(\frac{1-\mu}{r_{1}}+\frac{\mu}{r_{2}}\right)+\left(x^{2}+y^{2}\right)\right]
$$

We can reformulate the CRTBP into Port-Hamiltonian system description:

$$
\frac{\mathrm{d}}{\mathrm{d} t} \boldsymbol{x}=[\boldsymbol{J}-\boldsymbol{R}] \nabla_{x} H(\boldsymbol{x})+\boldsymbol{B} \boldsymbol{u}
$$

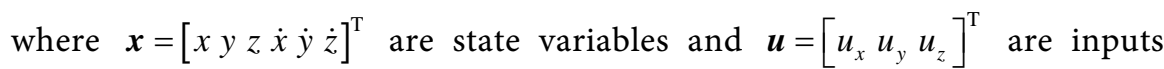
representing thrust force on $x, y$ and $z$ directions. In order to map inputs to state variables, we define $\boldsymbol{B}=\left[\begin{array}{cccccc}0 & 0 & 0 & 1 & 0 & 0 \\ 0 & 0 & 0 & 0 & 1 & 0 \\ 0 & 0 & 0 & 0 & 0 & 1\end{array}\right]^{\mathrm{T}} . J$ is a skew-symmetric matrix, which represents the energy conserving part, while matrix $R$ is a symmetric positive semi-definite matrix, which represents the energy dissipation:

$$
\boldsymbol{J}=\left[\begin{array}{cccccc}
0 & 0 & 0 & 1 & 0 & 0 \\
0 & 0 & 0 & 0 & 1 & 0 \\
0 & 0 & 0 & 0 & 0 & 1 \\
-1 & 0 & 0 & 0 & 2 & 0 \\
0 & -1 & 0 & -2 & 0 & 0 \\
0 & 0 & -1 & 0 & 0 & 0
\end{array}\right]^{\mathrm{T}}, \quad \boldsymbol{R}=0_{6 \times 6}
$$

Since CRTBP does not consider energy dissipation such as air drag, $R$ is a zero matrix. This causes the conservation of energy (Hamiltonian or Jacobian integral used here), which is the fact reflected in the following equation:

$$
\begin{aligned}
\frac{\mathrm{d} H(\boldsymbol{x})}{\mathrm{d} t} & =\nabla_{x} H(\boldsymbol{x}) \cdot \frac{\mathrm{d} x}{\mathrm{~d} t}=\nabla_{x} H(\boldsymbol{x}) \cdot[\boldsymbol{J}-\boldsymbol{R}] \nabla_{x} H(\boldsymbol{x}) \\
& =\nabla_{x} H(\boldsymbol{x}) \cdot[-\boldsymbol{R}] \nabla_{x} H(\boldsymbol{x})=0
\end{aligned}
$$

\subsection{Energy Shaping}

Instead of controlling the Hamiltonian, we are focused on reshaping the Hamil-

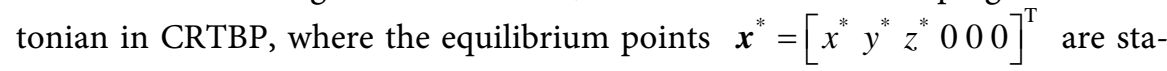
ble. Thus, we designed a closed-loop Hamiltonian $H_{d}(\boldsymbol{x})$ :

$$
H_{d}(x)=\frac{1}{2}\left[\dot{x}^{2}+\dot{y}^{2}+\dot{z}^{2}+\left(x-x^{*}\right)^{2}+\left(y-y^{*}\right)^{2}+\left(z-z^{*}\right)^{2}\right]
$$

with the minimal position:

$$
\boldsymbol{x}^{*}=\arg \min \left\{H_{d}(\boldsymbol{x})\right\}
$$

assuming that the closed loop system is also a Port-Hamilton system

$$
\frac{\mathrm{d} x}{\mathrm{~d} t}=[\boldsymbol{J}-\boldsymbol{R}] \nabla_{x} H_{d}
$$


Then, we design the feedback control law as $\boldsymbol{B}$ is full column rank but not full row rank:

$$
\begin{gathered}
\boldsymbol{U}_{E S}(\boldsymbol{x})=\left(\boldsymbol{B}^{\mathrm{T}} \boldsymbol{B}\right)^{-1} \boldsymbol{B}^{\mathrm{T}}[\boldsymbol{J}-\boldsymbol{R}] \nabla_{x} H_{a}(\boldsymbol{x}) \\
H_{a}(\boldsymbol{x})=H_{d}(\boldsymbol{x})-H(\boldsymbol{x})
\end{gathered}
$$

where the difference between close-loop system and open-loop system is shown by

$$
\boldsymbol{B}^{\perp}[\boldsymbol{J}-\boldsymbol{R}] \nabla_{x} H_{a}(\boldsymbol{x})=\mathbf{0}
$$

Under this control law, it can be shown that the closed-loop Hamiltonian is a Lyapunov function satisfying:

$$
\begin{gathered}
H_{d}(\boldsymbol{x})=0, \boldsymbol{x}=\boldsymbol{x}^{*} \\
H_{d}(\boldsymbol{x})>0, \forall \boldsymbol{x} \neq \boldsymbol{x}^{*} \\
\frac{\mathrm{d} H_{d}(\boldsymbol{x})}{\mathrm{d} t}=\nabla_{x} H_{d}(\boldsymbol{x}) \cdot[-\boldsymbol{R}] \nabla_{x} H_{d}(\boldsymbol{x})=0, \forall \boldsymbol{x} \neq \boldsymbol{x}^{*}
\end{gathered}
$$

According to Lyapunov stability theorem, the equilibrium $\boldsymbol{x}$ of the closed-loop system is stable.

\subsection{Dissipation Injection}

Previous energy shaping process utilizes a state feedback control law to shape the closed-loop Hamiltonian (energy) with a stable equilibrium at $\boldsymbol{x}^{*}$. However, the closed-loop Hamiltonian also remains a constant; i.e., $\frac{\mathrm{d} H_{d}(\boldsymbol{x})}{\mathrm{d} t}=\nabla_{x} H_{d}(\boldsymbol{x}) \cdot[-\boldsymbol{R}] \nabla_{x} H_{d}(\boldsymbol{x})=0$. We may also design the equilibrium point as asymptotically stable. This can be achieved through dissipation injection, which modifies the $R$ matrix in the closed-loop system. Aside from the energy shaping control law, we also implement a state feedback control representing dissipation injection:

$$
\boldsymbol{u}_{D I}(\boldsymbol{x})=-\boldsymbol{K}_{d} \boldsymbol{B}^{\mathrm{T}} \nabla_{x} H_{d}(\boldsymbol{x})
$$

where $\boldsymbol{K}_{d}$ is positive definite. With $\boldsymbol{u}(\boldsymbol{x})=\boldsymbol{u}_{E S}(\boldsymbol{x})+\boldsymbol{u}_{D I}(\boldsymbol{x})$, we have the closed-loop system as:

$$
\frac{\mathrm{d} x}{\mathrm{~d} t}=\left[\boldsymbol{J}-\boldsymbol{R}_{d}(\boldsymbol{x})\right] \nabla_{x} H_{d}
$$

with a closed-loop dissipation matrix: $\boldsymbol{R}_{d}(\boldsymbol{x})=\boldsymbol{R}(\boldsymbol{x})+\boldsymbol{B}(\boldsymbol{x}) \boldsymbol{K}_{d} \boldsymbol{B}^{\mathrm{T}}$.

Thus, the closed-loop Hamiltonian evolves like:

$$
\frac{\mathrm{d} H_{d}(\boldsymbol{x})}{\mathrm{d} t}=\nabla_{x} H_{d}(\boldsymbol{x}) \cdot\left[-\boldsymbol{R}_{d}\right] \nabla_{x} H_{d}(\boldsymbol{x}) \leq 0, \quad \forall \boldsymbol{x} \neq \boldsymbol{x}^{*}
$$

The closed-loop Hamiltonian $H_{d}(\boldsymbol{x})$ also satisfies $H_{d}(\boldsymbol{x})=0, \boldsymbol{x}=\boldsymbol{x}^{*}$ and $H_{d}(\boldsymbol{x})>0, \forall \boldsymbol{x}=\boldsymbol{x}^{*}$. 
As the union of complete trajectories contained entirely in the set has no trajectory except the trivial one $\boldsymbol{x}(t)=\mathbf{0}$ for $t \geq \boldsymbol{x}^{*}$, we obtain the asymptotic stability of the closed-loop system according to LaSalle's invariance principle [25]. The above framework of energy shaping and dissipation injection are summarized as Figure 1.

\section{Results and Discussion}

Figures 2-4 shows the orbit of the third body without background noise on $x-y$, $x-Z$, and $y-Z$ plane, respectively. The blue dashed lines in these figures indicated the controlled orbits using (a) Linear Quadratic Regulator (LQR) controller compared with (b) Port-Hamiltonian controller. The results show that the PortHamiltonian controller allows us to drive the third body to the target equilibrium position, while the LQR controller fail. The LQR controller is designed [11] [26] [27] according to the CRTBP linearized around the $L_{1}$ Lagrange point: $\frac{\mathrm{d} x}{\mathrm{~d} t}=\boldsymbol{A} \boldsymbol{x}+\boldsymbol{B u}$ where $\boldsymbol{A}$ is the Jacobian of $(\boldsymbol{J}-\boldsymbol{R}) \nabla H(\boldsymbol{x})$ evaluated at the equilibrium point $\boldsymbol{x}^{*}$; i.e. the first Lagrange point $L_{1}$ in this case. Using the Linear Quadratic Regulator (LQR) controller, we obtain the control law as $\boldsymbol{u}_{\mathrm{LQR}}=-\boldsymbol{K}\left(\boldsymbol{x}-\boldsymbol{x}^{*}\right)$, where $\boldsymbol{K}$ minimizes the cost function $J_{\mathrm{LQR}}={ }_{0}^{s_{0}^{\infty}} \boldsymbol{x}^{\mathrm{T}} \boldsymbol{Q} \boldsymbol{x}+\boldsymbol{u}^{\mathrm{T}} \boldsymbol{R} \boldsymbol{u}$ with $\boldsymbol{Q}=\boldsymbol{I}_{6 \times 6}$ and $\boldsymbol{R}=\boldsymbol{I}_{3 \times 3}$.

Figures 5-7 shows the orbit of the third body with background noise on $x-y$, $x-Z$, and $y-Z$ plane, respectively. This background noise is used to model the disturbance including eccentricity of the orbit, the gravity from the other bodies [14]. Especially, for the Sun-Earth Lagrange points mission, the perturbation from the Moon is critical. The results show that, under the background noise, the Linear Quadratic Regulator is not able to drive the third body to the target position; i.e., $L_{1}$ Lagrange point in the Sun-Earth CRTBP. However, the approach based on the Port-Hamiltonian successfully drive the third body to the targeting position.

Figure 8 \& Figure 9 show the time history of the controller inputs $u_{x}, u_{y}$ and $u_{z}$ without background noise and with background noise, respectively. Results indicate that the controller input for Port-Hamiltonian controller is asymptoting to zero as the third body is approaching the target location, in consistent with the observation of previous results. These results indicate that when the third body is approaching the target position or the disturbance is small, the required control input is also small.

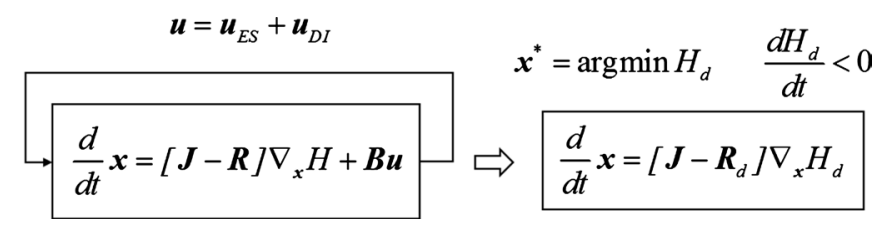

Figure 1. The flow chart of energy shaping and dissipation injection. Where $H(x)$ is a Hamiltonian, $H_{d}(\boldsymbol{x})$ is a closed-loop Hamiltonian, matrix $\boldsymbol{R}$ is a symmetric positive semi-definite matrix which represents the energy dissipation and $\boldsymbol{R}_{d}=\boldsymbol{R}+\boldsymbol{B}(\boldsymbol{x}) \boldsymbol{K}_{d} \boldsymbol{B}^{\mathrm{T}}$. 

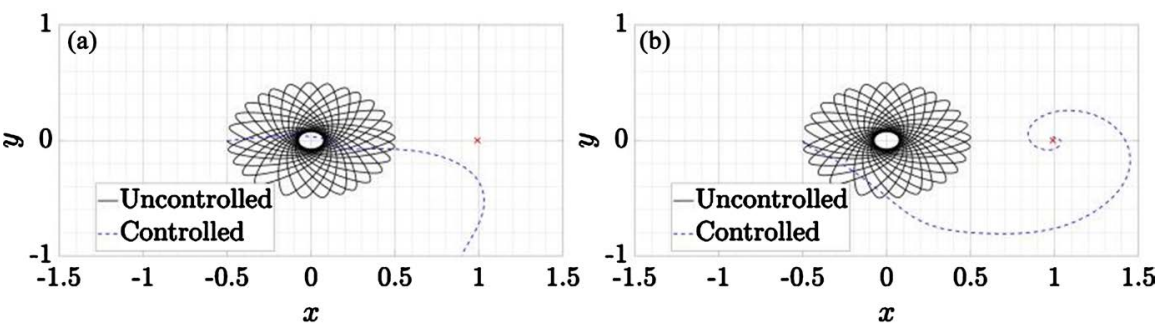

Figure 2. The orbit of the third body on $x-y$ plane without background noise. The black solid line represents the uncontrolled orbit, and the red cross represents $L_{1}$ Lagrange point. The blue dashed line represents the controlled orbit with (a) LQR controller and (b) port-Hamiltonian controller.
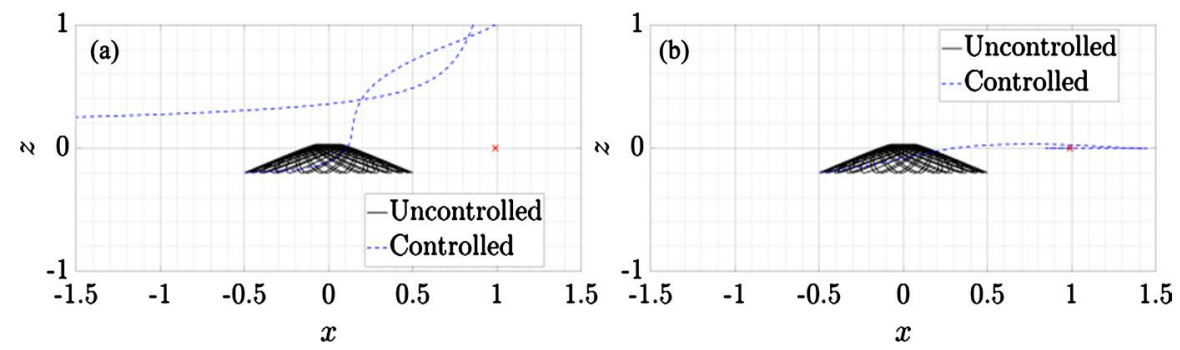

Figure 3. The orbit of the third body on $x-z$ plane without background noise. The black solid line represents the uncontrolled orbit, and the red cross represents $L_{1}$ Lagrange point. The blue dashed line represents the controlled orbit with (a) LQR controller and (b) port-Hamiltonian controller.


Figure 4. The orbit of the third body on $y-z$ plane without background noise. The black solid line represents the uncontrolled orbit, and the red cross represents $L_{1}$ Lagrange point. The blue dashed line represents the controlled orbit with (a) LQR controller and (b) port-Hamiltonina controller.


Figure 5. The orbit of the third body on $x-y$ plane with background noise, and the red cross represents $L_{1}$ Lagrange point. The black solid line represents the uncontrolled orbit; the blue dashed line represents the controlled orbit with (a) LQR controller compared with (b) port-Hamiltonian controller. 

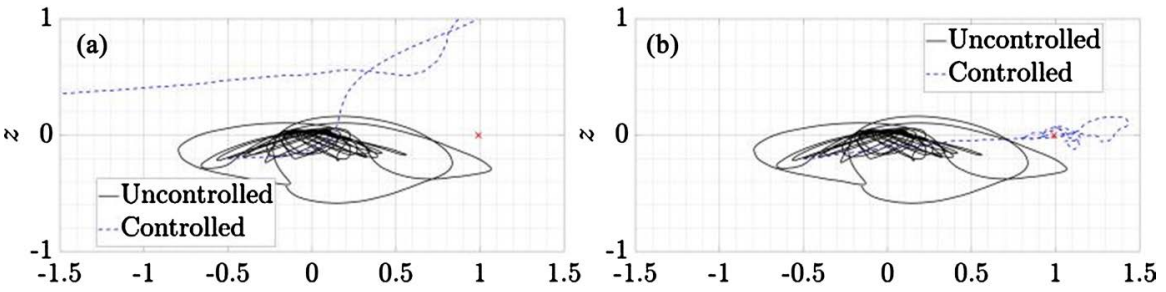

Figure 6. The orbit of the third body on $x$ - $z$ plane with background noise. The black solid line represents the uncontrolled orbit, and the red cross represents $L_{1}$ Lagrange point. The blue dashed line represents the controlled orbit with (a) LQR controller and (b) port-Hamiltonian controller.
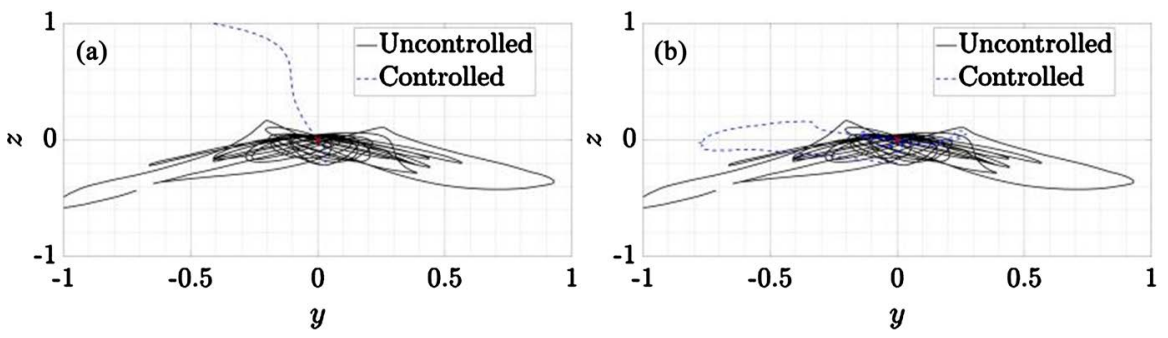

Figure 7. The orbit of the third body on $y$ - $z$ plane with background noise. The black solid line represents the uncontrolled orbit, and the red cross represents $L_{1}$ Lagrange point. The blue dashed line represents the controlled orbit with (a) LQR controller and (b) port-Hamiltonian controller.
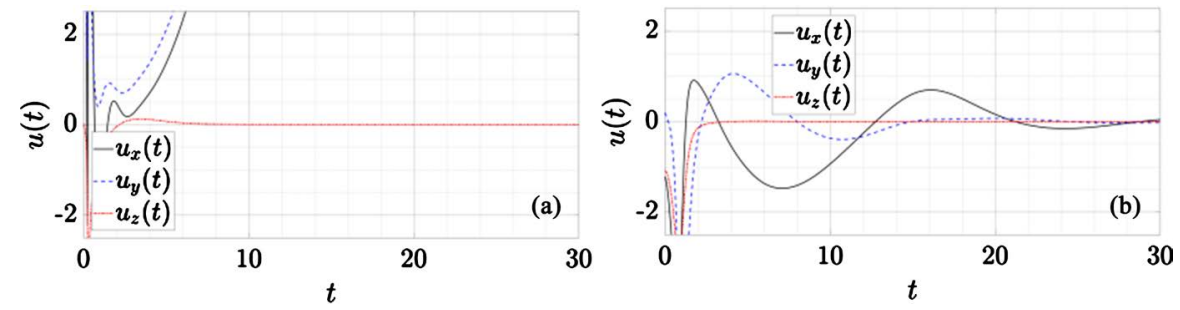

Figure 8. The control input according to the (a) LQR controller and (b) port-Hamiltonian controller without background noise.
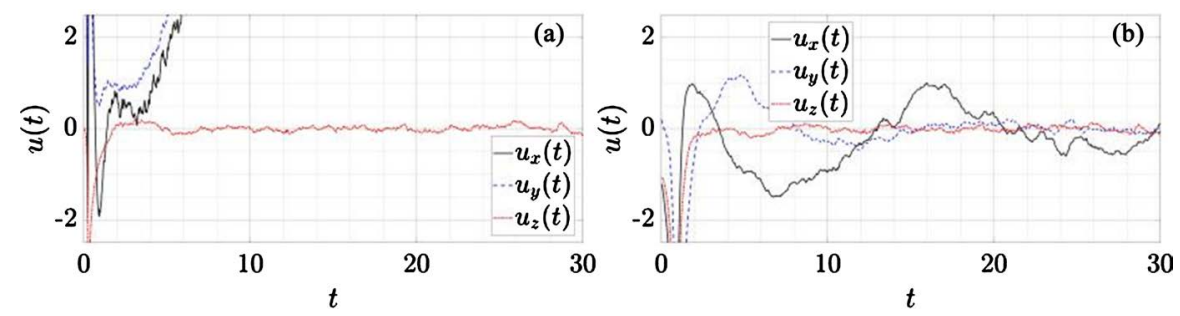

Figure 9. The control input according to the (a) LQR controller and (b) port-Hamiltonian controller with background noise.

\section{Conclusion}

In this paper, we use Port-Hamiltonian framework to stabilize the Lagrange points in the Circular Restricted Three-Body Problem (CRTBP). Through exploring the energy (Jacobi integral) conserving property in CRTBP, this problem is rewritten 
into Port-Hamiltonian framework, where we use the Jacobi integral as the Hamiltonian. Then, we design the feedback controller through energy-shaping and dissipation injection to stabilize the $L_{1}$ Lagrange point of the Sun-Earth CRTBP. The closed-loop Hamiltonian is designed as the candidate of the Lyapunov function to establish nonlinear stability of the designed equilibrium, which enlarges the application region of feedback controller compared with that based on linearized dynamics. Results show that the Port-Hamiltonian approach allows us to successfully stabilize the Lagrange points, where the Linear Quadratic Regulator (LQR) may fail. Adding the white noise into the inputs, the designed feedback controller based on Port-Hamiltonian approach also allows us to stabilize the Lagrange points, which demonstrates the robustness against the background noise of the designed feedback control.

\section{Acknowledgements}

Special thanks to Prof who offer us tremendous support and offer the idea of project. Special thanks to offering all the material, labor, technology, and all the equipment that our team needs to finish the project.

\section{Conflicts of Interest}

The author declares no conflicts of interest regarding the publication of this paper.

\section{References}

[1] Musielak, Z.E. and Quarles, B. (2014) The Three-Body Problem. Reports on Progress in Physics, 77, Article ID: 065901. https://doi.org/10.1088/0034-4885/77/6/065901

[2] Pilbratt, G.L., Riedinger, J.R., Passvogel, T., Crone, G., Doyle, D., Gageur, U., Heras, A.M., Jewell, C., Metcalfe, L., Ott, S., et al. (2010) Herschel Space Observatory. An ESA Facility for Far-Infrared and Submillimetre Astronomy. Astronomy \& Astrophysics, 518, Article No. L1. https://doi.org/10.1051/0004-6361/201014759

[3] Gardner, J.P., Mather, J.C., Clampin, M., Doyon, R., Greenhouse, M.A., Hammel, H.B., Hutchings, J.B., Jakobsen, P., Lilly, S.J., Long, K.S., et al. (2006) The James Webb Space Telescope. Space Science Reviews, 123, 485-606. https://doi.org/10.1007/s11214-006-8315-7

[4] Farquhar, R. (1998) The Flight of ISEE-3/ICE-Origins, Mission History, and a Legacy. AIAA/AAS Astrodynamics Specialist Conference and Exhibit, Boston, 10-12 August 1998, 4464. https://doi.org/10.2514/6.1998-4464

[5] Stone, E.C., Frandsen, A.M., Mewaldt, R.A., Christian, E.R., Margolies, D., Ormes, J.F. and Snow, F. (1998) The Advanced Composition Explorer. Space Science Reviews, 86, 1-22. https://doi.org/10.1023/A:1005082526237

[6] Sweetser, T.H., Broschart, S.B., Angelopoulos, V., Whiffen, G.J., Folta, D.C., Chung, M.K., Hatch, S.J. and Woodard, M.A. (2012) ARTEMIS Mission Design. In: Russell, C. and Angelopoulos, V., Eds., The ARTEMIS Mission, Springer, New York, 61-91. https://doi.org/10.1007/978-1-4614-9554-3 4

[7] Shirobokov, M., Trofimov, S. and Ovchinnikov, M. (2017) Survey of Station-Keeping Techniques for Libration Point Orbits. Journal of Guidance, Control, and Dynamics, 
40, 1085-1105. https://doi.org/10.2514/1.G001850

[8] Meyer, K.R. and Schmidt, D.S. (1986) The Stability of the Lagrange Triangular Point and a Theorem of Arnold. Journal of Differential Equations, 62, 222-236. https://doi.org/10.1016/0022-0396(86)90098-7

[9] Gómez, G., Jorba, A., Masdemont, J. and Simó, C. (1993) Study of the Transfer from the Earth to a Halo Orbit around the Equilibrium point $L_{1}$. Celestial Mechanics and Dynamical Astronomy, 56, 541-562. https://doi.org/10.1007/BF00696185

[10] Richardson, D.L. (1980) Halo Orbit Formulation for the ISEE-3 Mission. Journal of Guidance, Control, and Dynamics, 3, 543-548. https://doi.org/10.2514/3.56033

[11] Cielaszyk, D. and Wie, B. (1996) New Approach to Halo Orbit Determination and Control. Journal of Guidance, Control, and Dynamics, 19, 266-273. https://doi.org/10.2514/3.21614

[12] Ardaens, J.S. and D’Amico, S. (2008) Control of Formation Flying Spacecraft at a Lagrange Point. No. 00-08.

[13] Xu, M. and Xu, S.J. (2008) Trajectory and Correction Maneuver during the Transfer from Earth to Halo Orbit. Chinese Journal of Aeronautics, 21, 200-206. https://doi.org/10.1016/S1000-9361(08)60026-6

[14] Yamato, H. and Spencer, D. (2004) Transit-Orbit Search for Planar Restricted Three-Body Problems with Perturbations. Journal of Guidance, Control, and Dynamics, 27, 1035-1045. https://doi.org/10.2514/1.4524

[15] Lyapunov, A.M. (1992) The General Problem of the Stability of Motion. International Journal of Control, 55, 531-534. https://doi.org/10.1080/00207179208934253

[16] Jacobi, C.G.J. (1836) Sur le mouvement d'un point et sur un cas particulier du probleme destrois corps. Comptes Rendus Chimie, 3, 59-61.

[17] Van Der Schaft, A.J. and Schumacher, J.M. (2000) An Introduction to Hybrid Dynamical Systems. Springer, London, 251. https://doi.org/10.1007/BFb0109998

[18] Van Der Schaft, A.J., Jeltsema, D. et al. (2014) Port-Hamiltonian Systems Theory: An Introductory Overview. Foundations and Trends in Systems and Control, 1, 173-378. https://doi.org/10.1561/2600000002

[19] Ortega, R., Van Der Schaft, A.J., Mareels, I. and Maschke, B. (2001) Putting Energy Back in Control. IEEE Control Systems Magazine, 21, 18-33. https://doi.org/10.1109/37.915398

[20] Ortega, R., Van Der Schaft, A.J., Maschke, B. and Escobar, G. (2002) Interconnection and Damping Assignment Passivity-Based Control of Port-Controlled Hamiltonian Systems. Automatica, 38, 585-596. https://doi.org/10.1016/S0005-1098(01)00278-3

[21] Ortega, R., Van Der Schaft, A.J., Castanos, F. and Astolfi, A. (2008) Control by Interconnection and Standard Passivity-Based Control of Port-Hamiltonian Systems. IEEE Transactions on Automatic Control, 53, 2527-2542. https://doi.org/10.1109/TAC.2008.2006930

[22] Liu, C. (2019) Teaching Control Theory in Physics: The Port-Hamiltonian Framework. College Physics, 38, 1-7.

[23] Liu, C. and Dong, L. (2019) Physics-Based Control Education: Energy, Dissipation, and Structure Assignments. European Journal of Physics, 40, Article ID: 035006. https://doi.org/10.1088/1361-6404/ab03e8

[24] Liu, C. and Dong, L. (2019) Stabilization of Lagrange Points in Circular Restricted Three-Body Problem: A Port-Hamiltonian Approach. Physics Letters A, 383, 1907-1914. https://doi.org/10.1016/j.physleta.2019.03.033 
[25] LaSalle, J. (1960) Some Extensions of Liapunov's Second Method. IRE Transactions on Circuit Theory, 7, 520-527. https://doi.org/10.1109/TCT.1960.1086720

[26] Kwakernaak, H. and Sivan, R. (1972) Linear Optimal Control Systems. Wiley-Interscience, New York.

[27] Zhou, K., Doyle, J.C., Glover, K., et al. (1996) Robust and Optimal Control. Prentice Hall, Upper Saddle River. 